\title{
不同生育时期玉米洪涝胁迫遥感监测与评估
}

\author{
隋学艳 ${ }^{1, * *}$ 梁守真 ${ }^{1, * *}$ 张金盈 $^{2}$ 王 猛 ${ }^{1}$ 王 勇 ${ }^{1}$ 侯学会 ${ }^{1}$ \\ 张晓冬 3 , \\ ${ }^{1}$ 山东省农业可持续发展研究所 / 农业农村部华东都市农业重点实验室, 山东济南 $250100 ;{ }^{2}$ 山东省国土测绘院, 山东济南 250013; \\ ${ }^{3}$ 山东省农作物种质资源中心，山东济南 250100
}

\begin{abstract}
摘 要: 为建立玉米洪涝灾害遥感监测和评估技术, 本研究开展不同生育期不同程度的模拟试验, 持续活体监测叶片 叶绿素、冠层光谱和覆盖度并测产。结果表明, 拔节期洪涝胁迫可显著降低叶绿素含量, 最大程度相对变化值达 $-56.30 \%$, 吐丝期胁迫对叶绿素无影响, 灌浆期胁迫促使叶绿素下降但并不明显。洪涝胁迫能降低覆盖度, 拔节期影响最大, 胁迫 严重的覆盖度仅 $46.33 \%$ ，吐丝期次之，灌浆期较小。洪涝胁迫造成的减产，生育前期影响比后期严重。拔节期和吐丝期 各波段反射率与洪涝胁迫程度呈负相关关系，近红外平台波段达到极显著水平，绿峰波段次之，达到显著水平。灌浆期 各波段反射率与洪涝胁迫程度呈正相关关系，相关性未达到显著水平。与胁迫程度呈极显著相关的光谱反射率和植被指 数可以用于监测玉米洪涝灾害。最终基于胁迫之后与胁迫前的结构不敏感色素指数 SIPI 的差值 DSIPI, 建立拔节期和吐 丝期洪涝胁迫产量损失率评估模型。
\end{abstract}

关键词: 玉米; 涝灾; 遥感; 监测; 评估

\section{Remote sensing monitoring on maize flood stress and yield evaluation at different stages}

\author{
SUI Xue-Yan ${ }^{1, * *}$, LIANG Shou-Zhen ${ }^{1, * *}$, ZHANG Jin-Ying ${ }^{2}$, WANG Meng ${ }^{1}$, WANG Yong ${ }^{1}$, HOU Xue-Hui ${ }^{1}$, \\ and ZHANG Xiao-Dong ${ }^{3, *}$

\footnotetext{
${ }^{1}$ Shandong Institute of Agricultural Sustainable Development / Key Laboratory of East China Urban Agriculture, Ministry of Agriculture and Shandong Rural Affairs, Jinan 250100, Shandong, China; ${ }^{2}$ Shandong Provincial Institute of Land Surveying and Mapping, Jinan 250013, Shandong, China; ${ }^{3}$ Shandong Center of Crop Germplasm Resources, Jinan 250100, Shandong, China
}

\begin{abstract}
In order to set up remote sensing monitoring and evaluation technology of maize flood stress and loss, a simulation experiment with different flood stress degrees was developed at different stages. Chlorophyll content, canopy spectral reflectance and coverage were monitored in vivo, and yield level was tested finally. The results showed that chlorophyll content decreased under flood stress at jointing and filling stages. The decreasing extend was significant at jointing stage and the value of maximal relative change reached $-56.30 \%$. There was little influence on chlorophyll at silking stage. Flood stress could reduce the coverage, especially at jointing stage, the most serious treatment was only $46.33 \%$, followed by silking stage and filling stage. Flood stress reduced production at last, and the reduction was more serious in the early stage than in the later stage. There was a negative correlation between reflectance and flood stress degree at jointing stage and silking stage. It was extremely significantly difference at near infrared platform bands and significantly at green peak bands. There was no significant positive correlation between reflectance and flood stress degree at filling stage. Reflectance and spectral indexes with extremely significant correlation can be used to monitor flood. Two models with DSIPI were set up to evaluate flood loss at jointing and silking stage separately.
\end{abstract}

Keywords: maize; flood stress; remote sensing; monitoring; evaluation

\footnotetext{
本研究由山东省农业重大应用技术创新项目(1-0504)和农业农村部农业农村资源监测统计项目(061721301112422018)资助。

This study was supported by the Agricultural Great Application Technology Innovative Projects of Shandong Province (1-0504) and the Agricultural and Rural Resources Monitoring and Statistics Projects of Ministry of Agriculture and Rural Affairs (061721301112422018).

*通信作者(Corresponding author): 张晓冬, E-mail: zxdong2002@163.com

** 同等贡献(Contributed equally to this work)

第一作者联系方式: 隋学艳, E-mail: sdnkysxy@163.com; 梁守真, E-mail: szliang_cas@163.com

Received (收稿日期): 2020-02-28; Accepted (接受日期): 2020-08-20; Published online (网络出版日期): 2020-09-10.

URL: https://kns.cnki.net/kcms/detail/11.1809.S.20200909.1037.004.html
} 
玉米是我国主要的粮食作物, 其用途较其他粮食作 物广泛 ${ }^{[1]}, 2018$ 年玉米播种面积占全国粮食播种面积的 $36.0 \%$, 位居粮食作物播种面积首位 ${ }^{[2]}$ 。玉米属于 $\mathrm{C}_{4}$ 作物, 与 $\mathrm{C}_{3}$ 作物相比具有较强的抗旱能力和抗热能力 ${ }^{[3]}$, 需水 量大而不耐涝 ${ }^{[4]}$ 。我国玉米生育时期多集中在 6 月至 9 月, 频繁的强对流天气致使玉米遭受洪涝灾害, 受灾后玉米

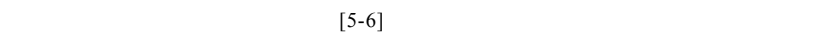
失调、光合能力降低 ${ }^{[7]}$ 、新叶片出生速率降低老叶片变黄 ${ }^{[8]}$ 、 叶面积指数降低 ${ }^{[9]}$ 、叶绿素含量和氮素降低 ${ }^{[10]}$, 生育时期 延迟 ${ }^{[11]}$ 、产量下降 ${ }^{[12]}$ 。暴雨过后各级生产管理部门需要 尽快掌握玉米洪涝灾害发生的面积和程度, 制定合理的 救灾和减灾措施 ${ }^{[13]}$, 同时农业保险部门也要争分夺秒调 查灾情, 做好理赔工作。当前洪涝灾害的调查程序为: 农 户上报, 行政部门逐级汇总统计, 保险公司实地核查并组 织农业专家对灾损进行评估。暴雨发生时会造成道路损 坏、桥梁坞塌, 车辆无法通行, 玉米多为规模化种植, 地 块大、植株高, 靠人力很难在短时间内实现全面地、具有 地理属性的清查。玉米涝灾的发生发展, 是植株逆境下进 行生理生化反应的动态变化过程 ${ }^{[3]}$, 数据的准确性和科学 性是灾害救助决策方案制定的重要保障。以遥感为主的空 间信息技术是当前快速、准确、大面积获取地面信息的手 段 ${ }^{[14]}$, 遥感技术已被广泛地应用于洪水淹没区域监测 ${ }^{[15-17]}$, 和 玉米长势 ${ }^{[18]}$ 与产量 ${ }^{[19-20]}$ 评估研究, 但在玉米洪涝灾害监 测与评估方面的研究鲜有报道。本研究采用人工模拟的办
法, 观测不同生育时期、不同程度洪涝胁迫玉米冠层光谱 及长势变化, 旨在建立玉米洪涝灾害遥感监测与灾损评 估的技术方法。

\section{1 材料与方法}

\section{1 试验设计}

洪涝胁迫试验于 2015 年进行, 地点在山东省济南市 济阳县太平镇姜家村 $\left(39^{\circ} 57.822^{\prime} \mathrm{N}, 116^{\circ} 19.602^{\prime} \mathrm{E}\right)$, 胁迫实 验设置早(拔节期)、中(吐丝期)、晚(灌浆期) 3 个时期, 每 个时期设 $0 、 1 、 3 、 5 、 7$ 和 $9 \mathrm{~d} 6$ 个胁迫水平, 其中 $0 \mathrm{~d}$ 为对照。小区长 $6.0 \mathrm{~m}$, 宽 $2.5 \mathrm{~m}$, 共 18 个小区, 玉米行距 $0.6 \mathrm{~m}$, 株距 $30 \mathrm{~cm}$, 玉米品种为登海 605 。小区四周铺埋 塑料布, 筑高 $0.4 \mathrm{~m}$ 、宽 $0.5 \mathrm{~m}$ 土堰, 防止水分外渗, $24 \mathrm{~h}$ 持续注水, 水深保持 $30 \mathrm{~cm}$ 。胁迫处理情况见表 1 。

1.2 测定项目及方法

1.2.1 冠层高光谱测量 自 7 月 31 日拔节期胁迫处理 开始, 天气晴朗日持续进行冠层光谱测量, 直至灌浆中后 期。采用美国 ASD Field SpecHandHeld 野外便携式高光 谱仪进行光谱测量, 测量时间为北京时间 11:00-14:00。 光纤探头垂直向下固定于自制观测架的横臂上, 架子高 $3.4 \mathrm{~m}$, 横臂长 $0.75 \mathrm{~m}$ 。每小区用插地标牌固定南中北 3 个测试点, 测试前进行白板标定, 每点采集 1 条光谱, 取 均值作为测量结果。

1.2.2 覆盖度测量 光谱观测架横臂紧临光纤探头处

表 1 试验设计

Table 1 Experimental design

\begin{tabular}{ccccc}
\hline $\begin{array}{c}\text { 生长时期 } \\
\text { Growing stage }\end{array}$ & $\begin{array}{c}\text { 处理 } \\
\text { Treatment }\end{array}$ & $\begin{array}{c}\text { 胁迫持续天数 } \\
\text { Flooding period (d) }\end{array}$ & $\begin{array}{c}\text { 开始时间 } \\
\text { Starting time (month/day) }\end{array}$ & $\begin{array}{c}\text { 结束时间 } \\
\text { Ending time (month/day) }\end{array}$ \\
\hline 拔节期 & E-9 & 9 & $7 / 31$ & $8 / 9$ \\
Jointing stage & E-7 & 7 & $7 / 31$ & $8 / 7$ \\
& E-5 & 5 & $7 / 31$ & $8 / 5$ \\
& E-3 & 3 & $7 / 31$ & $8 / 3$ \\
E-1 & 1 & $7 / 31$ & $8 / 1$ \\
吐丝期 & E-0 & $0(\mathrm{CK})$ & & $8 / 17$ \\
Silking stage & M-9 & 9 & $8 / 8$ & $8 / 15$ \\
& M-7 & 7 & $8 / 8$ & $8 / 13$ \\
& M-5 & 5 & $8 / 8$ & $8 / 11$ \\
& M-3 & 3 & $8 / 8$ & $8 / 9$ \\
M-1 & 1 & $8 / 8$ & \\
灌浆期 & M-0 & $0(\mathrm{CK})$ & & $9 / 1$ \\
Filling stage & L-9 & 9 & $8 / 23$ & $8 / 30$ \\
& L-7 & 7 & $8 / 23$ & $8 / 28$ \\
& L-5 & 5 & $8 / 23$ & $8 / 26$ \\
& L-3 & 3 & $8 / 23$ & $8 / 24$ \\
\hline
\end{tabular}


垂直悬挂照相机 SAMSUNG 100II, 调整焦距, 使拍摄范 围与光谱测试范围相同, 与光谱同步拍摄, 用图像处理软 件计算绿色占整幅图像的面积百分比得覆盖度, 取均值 作为测量结果。

1.2.3 叶绿素测量 每小区选取长势均匀的连续 10 株玉米, 固定叶片用植物养分测定仪 TYS - 3N 活体监测。 拔节期选取玉米植株最上部第 1 片完全展开叶, 吐丝期 和灌浆期选定玉米穗上部第 1 片叶片, 测试时间与光谱 测试同步。

1.2.4 产量测定蜡熟期全部收获，每行玉米作为 1 个样本, 测定产量构成因素; 晾晒后脱粒称重, 并用 PM-8188-A 谷物水分测量仪随机测定 3 次玉米含水量。折 算每公顷穗数、平均穗粒数、千粒重以及每公顷产量。

1.2.5 数据处理 根据试验采集数据情况, 统一选取每 个时期全部胁迫处理完后 $5 \mathrm{~d}$ 与洪涝胁迫之前的测试数据 进行分析。采用 ASD ViewSpecPro 软件对光谱数据进行初
处理; 采用 ENVI、MATLAB 图像处理软件提取覆盖度; 采 用 Microsoft Excel 软件进行数据整理与作图; 采用 DPS 软 件进行数据统计分析和差异显著性检验(Duncan's 法)。

\section{2 结果与分析}

\section{1 洪涝胁迫对叶绿素的影响}

洪涝胁迫后被观测叶片叶绿素呈现复杂的变化现象。 由图 1 可见, 拔节期洪涝胁迫对照处理 E- 0 和 E- 1 的最上 部第 1 片展开叶的叶绿素稍有增加, 其他处理的叶绿素均 降低, 处理 E-9 和 E-7 极显著低于其他处理, 处理 E-9 胁 迫后叶片叶绿素降至 17.55, 仅为之前的 $45.70 \%$; 吐丝期 玉米棒上第 1 片叶片的叶绿素只有 M-9 处理下降, 其他处 理均增加, 增加幅度无明显规律, 洪涝胁迫对被观测叶片 的叶绿素无影响; 灌浆期玉米处于生育后期, 叶片逐渐衰 老, 叶绿素含量会逐渐降低, 洪涝胁迫促使叶绿素下降但 并不明显。

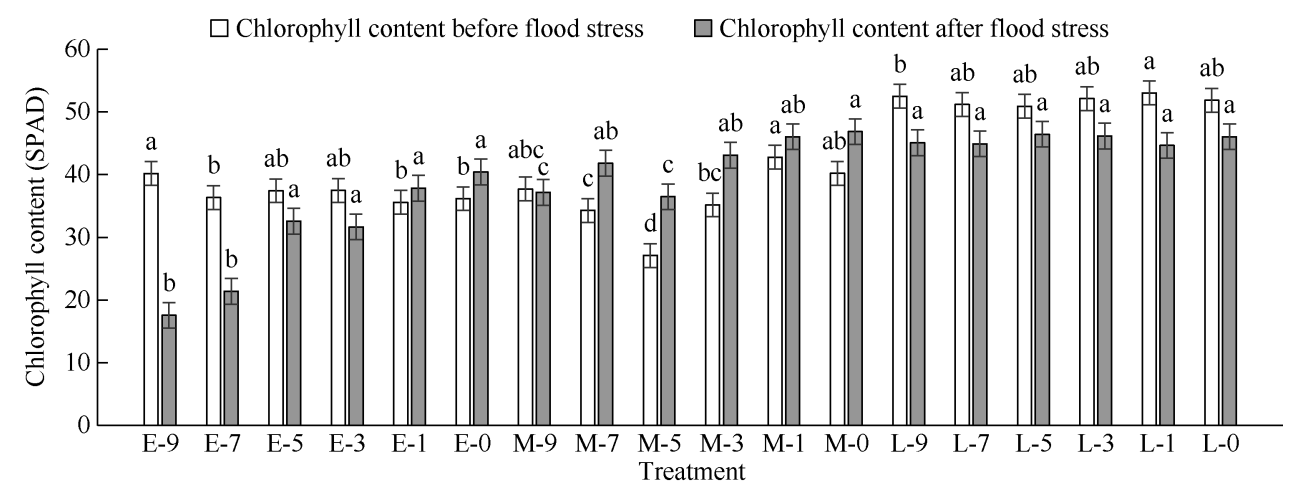

图 1 洪涝胁迫前后玉米叶绿素含量

Fig. 1 Chlorophyll content before and after flood stress in maize 处理同表 1 。不同字母表示同期处理间在 0.05 水平差异显著。

Treatments are the same as those given in Table 1. Different letters indicate significant differences among treatments at the 0.05 probability level.

\section{2 洪涝胁迫对覆盖度的影响}

洪涝胁迫能够降低玉米覆盖度, 玉米全生育期内拔 节期覆盖度最高, 吐丝期次之, 灌浆期最低。处理之前各 小区覆盖度均匀, 胁迫之后处理间覆盖度部分达到了极 显著的差异水平。3 个生育时期洪涝胁迫均能降低玉米覆 盖度, 以拔节期影响最大, 相对变化值最高幅度为 $-40.67 \%$, 吐丝期次之, 灌浆期较小(图 2)。

\section{3 洪涝胁迫对玉米冠层光谱的影响}

2.3.1 洪涝胁迫对反射率的影响植物体内因含有叶 绿素、水、蛋白质等复杂的化学组分, 以及多层叶片叠 交的冠层结构而产生不同于其他地物的光谱反射率曲 线 ${ }^{[21]}$ 。选择 3 个时期洪涝胁迫处理完成 $5 \mathrm{~d}$ 后的小区冠 层光谱, 绘制光谱曲线(图 3 图 5)。与对照 E-0 相比, 拔 节期洪涝胁迫, 造成原有叶片叶绿素含量降低, 下部叶 片迅速衰老, 新生叶片生长速度降低, 覆盖度降低, 露 出植株底部土壤, 土壤及土壤中的水分对太阳光可见光
至近红外的吸收要高于植被, 因此整体呈现反射率降低 的现象，胁迫程度越高降低越大，可见光波段 $553 \mathrm{~nm}$ 处反射率降低幅度最大, 为 0.022 , 近红外平台 $879 \mathrm{~nm}$ 处反射率降低幅度最大, 为 0.340 (图 3)。吐丝期玉米植 株的叶片数量已经基本确定, 最后几片新生叶片逐渐展 开, 洪涝胁迫主要造成下部叶片的衰老, 降低了新生叶 片展开的速度, 胁迫处理与对照 M- 0 相比光谱反射率在 可见光波段降低，最大幅度在 $553 \mathrm{~nm}$ 处 0.016 , 在近红 外波段反射率同样下降，最大幅度在 $879 \mathrm{~nm}$ 处 0.199 , 整体趋势同拔节期洪涝胁迫一致, 但降低幅度均变小 (图 4)。灌浆期洪涝胁迫处理 L-7、L-5 和 L-9 与对照 L-0 的反射率相比可见光有明显的升高, 处理 L-5 和 L-7 与 对照 L-0 的反射率相比近红外有明显的升高, 处理 L-9 与对照 L-0 的反射率相比近红外波段基本一致, 处理 L-3、L-1 与对照 L-0 的反射率相比, 在可见光和近红外 均有明显的降低(图 5)。 


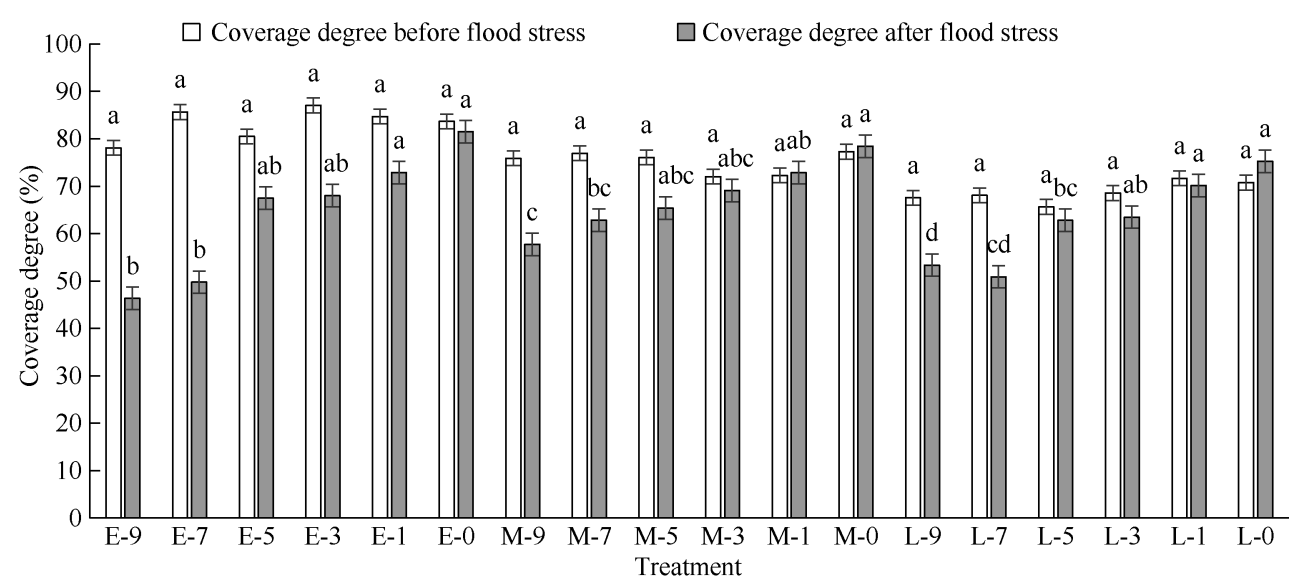

图 2 洪涝胁迫对玉米覆盖度的影响

Fig. 2 Effects of water stress on maize coverage degree

处理同表 1 。不同字母表示同期处理间在 0.05 水平差异显著。

Treatments are the same as those given in Table 1. Values marked with different letters indicate significant differences among treatments at the 0.05 probability level.

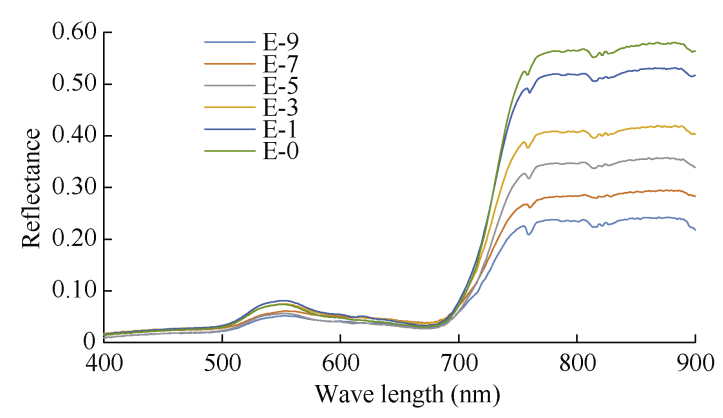

图 3 拔节期洪涝胁迫各处理 8 月 14 日光谱反射率曲线

Fig. 3 Spectral reflectance curves of flooding stress treatments at jointing stage on August 14

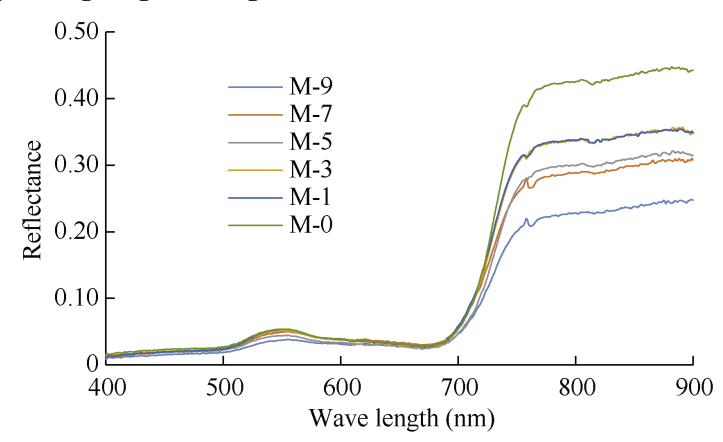

图 4 吐丝期洪涝胁迫各处理 8 月 23 日光谱反射率曲线

Fig. 4 Spectral reflectance curves of flooding stress treatments at silking stage on August 23

进一步对 3 个时期洪涝胁迫后光谱反射率与胁迫天 数进行相关性分析, 绘制曲线图。拔节期和吐丝期的胁迫 天数与光谱反射率相关性曲线形状比较相近, 即 400 900 nm 基本呈负相关关系, 整体分为 3 部分, 在蓝绿 光波段相关度较高, 在红谷波段相关度较低, 在近红外波 段相关度较高。由于胁迫时期不同, 细节上仍然存在明显 不同，拔节期仅在较窄的可见光 508 567 $\mathrm{nm}$ 的绿峰波段 达到了显著相关水平，而吐丝期在较宽的可见光 400 564 nm 的蓝光吸收谷和绿光反射峰都达到了显著相

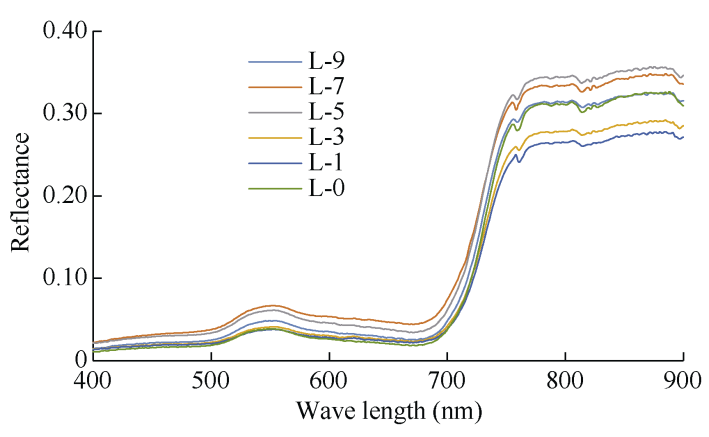

图 5 灌浆期洪涝胁迫各处理 9 月 3 日光谱反射率曲线

Fig. 5 Spectral reflectance curves of flooding stress treatments at filling stage on September 3

关关系。拔节期在较宽的 711 900 nm 波段达到了极显著 相关关系, 吐丝期在较窄的 727 896 nm 波段达到了极显 著相关关系。与拔节期和吐丝期不同的是，灌浆期的胁迫 天数与光谱反射率在 400 900 $\mathrm{nm}$ 均呈正相关关系, 即胁 迫天数越长, 各波段的光谱反射率越高(图 6)。

\subsection{2 洪涝胁迫对光谱形状参数和植被指数的影响}

洪涝胁迫对叶绿素含量、冠层结构的影响, 改变光谱

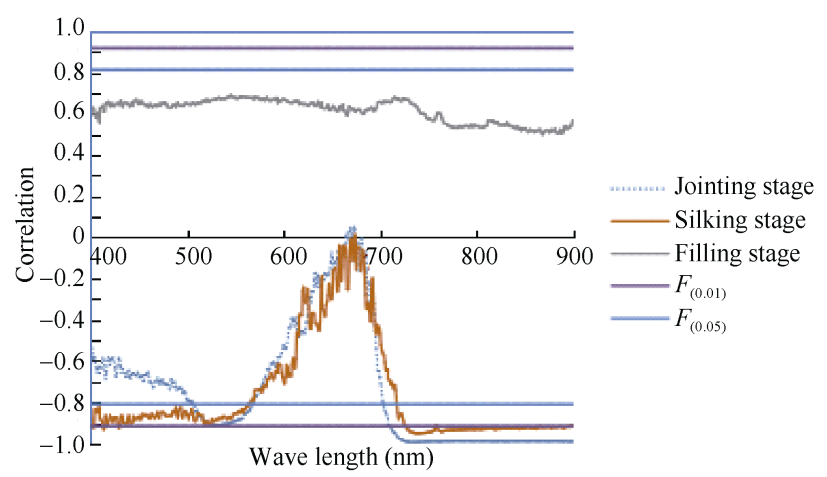

图 6 各时期洪涝胁迫后光谱反射率与胁迫天数的相关性

Fig. 6 Correlation between spectral reflectance and flooding stress period of treatments at different stages 
反射率的同时也改变了光谱曲线的形状。计算拔节期、吐 丝期、灌浆期的洪涝胁迫后经典光谱形状参数和植被指数
共 25 个, 并计算 25 个指数与洪涝胁迫天数的相关性, 结 果见表 2 。

表 2 洪涝胁迫天数与光谱形状参数和植被指数的相关性

Table 2 Correlation of flooding stress period with spectral shape parameters and vegetation indexes

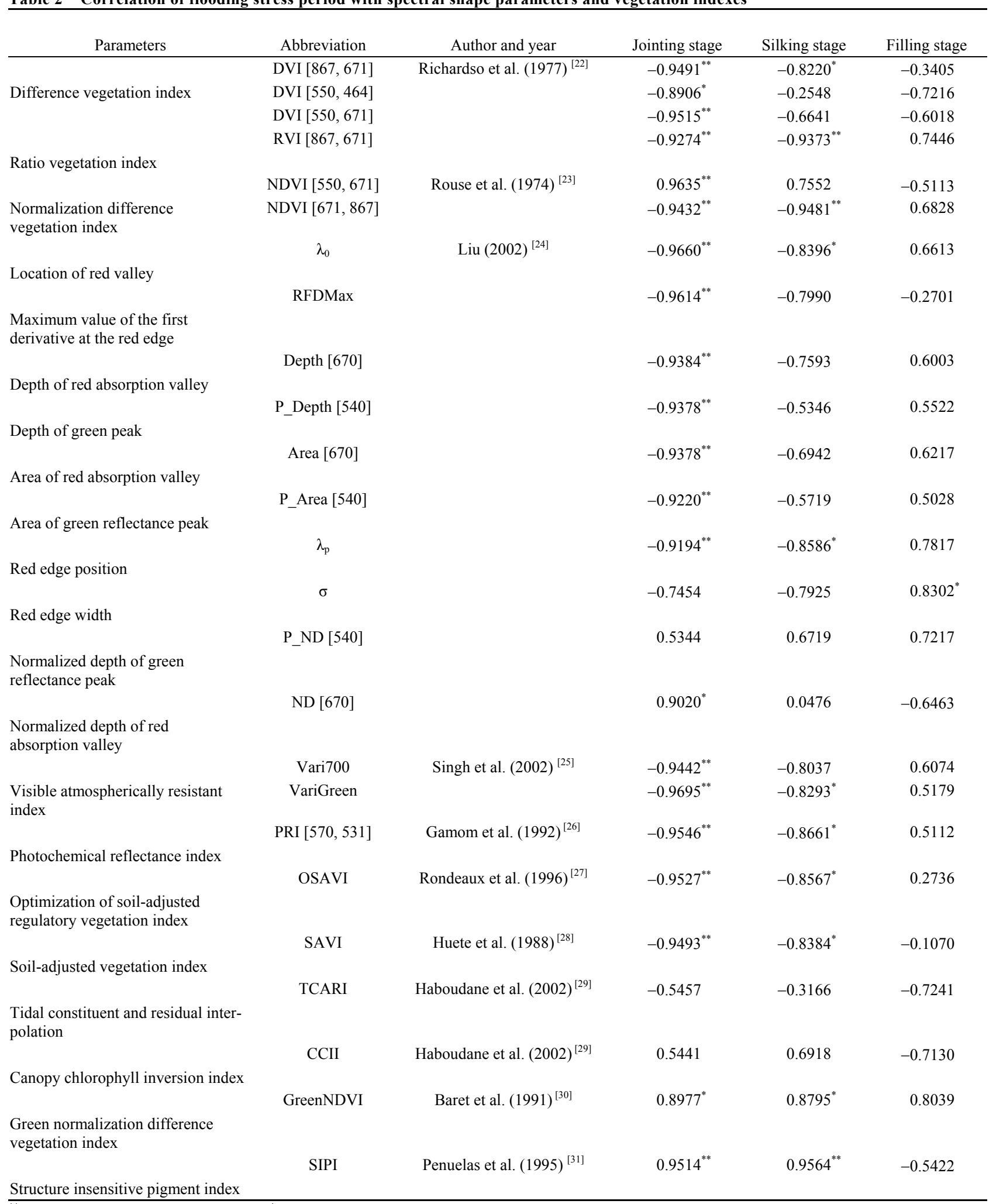

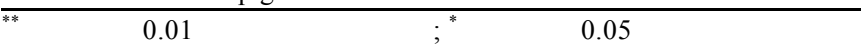

${ }^{* *}$ and ${ }^{*}$ indicate significance at the 0.01 and 0.05 probability levels, respectively. 
洪涝胁迫天数与光谱形状参数和植被指数的相关性 依然是拔节期和吐丝期较大, 灌浆期最小, 且以负相关为 主。25 个指数中拔节期 17 个达到极显著相关水平, 4 个达 到显著相关水平; 吐丝期 3 个达到极显著相关水平, 8 个 达到显著相关水平; 灌浆期 1 个达到显著相关水平。归一 化差值植被指数 NDVI [671,867]、比值植被指数 RVI [867, 671]、结构不敏感色素指数 SIPI 在拔节期和吐丝期的洪 涝胁迫程度均达到极显著相关水平, 可以作为 2 个生育时
期洪涝灾害监测的共同指数。

\section{4 洪涝胁迫玉米产量损失评估}

将胁迫处理完后 $5 \mathrm{~d}$ 与洪涝胁迫之前的归一化差值植 被指数 NDVI [671, 867]、比值植被指数 RVI [867, 671]、 结构不敏感色素指数 SIPI 的差值 DNDVI、DRVI、DSIPI 作为洪涝胁迫产量损失率估算的参数, 分别对拔节期和 吐丝期的洪涝胁迫玉米产量损失率进行线性、多项式拟合, 拟合效果均以多项式较优(表 3)。

表 3 拔节期和吐丝期洪涝胁迫玉米产量损失率模型

Table 3 Model of maize yield loss rate under flooding stress at jointing stage and silking stage

\begin{tabular}{|c|c|c|c|}
\hline $\begin{array}{c}\text { 参数 } \\
\text { Parameter }\end{array}$ & $\begin{array}{l}\text { 参数计算公式 } \\
\text { Parameter calculation } \\
\text { formula } \\
\end{array}$ & $\begin{array}{c}\text { 拔节期洪涝胁迫玉米产量损失率模型 } \\
\text { Model of maize yield loss rate under flooding stress at } \\
\text { jointing stage } \\
\text {. }\end{array}$ & $\begin{array}{l}\text { 吐丝期洪涝胁迫玉米产量损失率模型 } \\
\text { Model of maize yield loss rate under flood- } \\
\text { ing stress at silking stage } \\
\end{array}$ \\
\hline DNDVI & $\mathrm{NDVI}_{\text {after }}-\mathrm{NDVI}_{\text {before }}$ & $\begin{array}{c}y=-86.051 x^{2}+470.53 x+9.5564 \\
(-0.04<x<0.14,0<y<75 \%) \\
R^{2}=0.8969\end{array}$ & $\begin{array}{c}y=-2803.8 x^{2}-586.71 x+13.8 \\
(-0.07<x<0.03,0<y<45 \%) \\
R^{2}=0.9337\end{array}$ \\
\hline DRVI & $\mathrm{RVI}_{\text {after }}-\mathrm{RVI}_{\text {before }}$ & $\begin{array}{c}y=-1163.9 x^{2}-855.71 x+9.8187 \\
(-0.09<x<0.02,0<y<75 \%) \\
R^{2}=0.8951\end{array}$ & $\begin{array}{c}y=-9118.2 x^{2}+1024.7 x+13.888 \\
(-0.02<x<0.05,0<y<45 \%) \\
R^{2}=0.9320\end{array}$ \\
\hline DSIPI & $\mathrm{SIPI}_{\text {after }}-\mathrm{SIPI}_{\text {before }}$ & $\begin{array}{c}y=-19600 x^{2}-1985.3 x+15.814 \\
(-0.06<x<0.01,0<y<75 \%) \\
R^{2}=0.9433\end{array}$ & $\begin{array}{c}y=-13599 x^{2}-814.11 x+26.53 \\
(-0.03<x<0.03,0<y<45 \%) \\
R^{2}=0.9697\end{array}$ \\
\hline
\end{tabular}

DNDVI 为归一化差值植被指数的差值, DRVI 为比值植被指数的差值, DSIPI 为结构不敏感色素指数的差值。 after 表示洪涝胁迫 $5 \mathrm{~d}$ 后, before 表示洪涝胁迫前。

DNDVI is the difference of NDVI; DRVI is the difference of RVI; DSIPI is the difference of SIPI. The "after" indicates the time five days after flooding stress, and the "before" indicates the time before flooding stress.

3 个参数 DNDVI、DRVI、DSIPI 分别建立的多项式 产量损失率评估模型, 以 DSIPI 为参数建立的模型效果最 好, 模型拟合图见图 7 和图 8, 其次为参数 DNDVI 建立的 产量损失评估模型, 再次为参数 DRVI 建立的模型。

\section{3 讨论}

玉米洪涝灾害是玉米生产中面临的重要气象灾害, 生产单位和政府于灾后均需及时掌握受灾面积和受损程 度。当前已有玉米洪涝胁迫后玉米生理变化的深入研究, 以及以水体识别为主的洪涝灾害面积遥感监测的研究和 应用, 但将玉米胁迫生理变化同遥感监测与评估技术相 结合的相关研究鲜有报道, 玉米洪涝灾害监测仍停留在

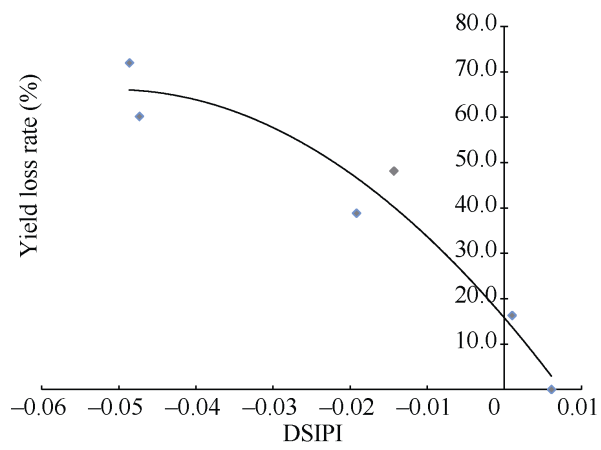

图 7 拔节期玉米洪涝胁迫产量损失评估 DSIPI 模型拟合图 Fig. 7 Fitting chart of maize yield loss rate with DSIPI under flooding stress at jointing stage
定性识别的阶段。为了建立玉米洪涝胁迫遥感监测和灾损 评估方法，本研究克服玉米洪涝灾害突发性和不可重复 性的特点，系统开展不同生育时期、不同胁迫程度玉米洪 涝灾害人工模拟试验，活体、持续、定位监测叶片叶绿素、 冠层光谱和覆盖度, 分析洪涝胁迫下叶绿素、覆盖度的变 化，从典型植被光谱曲线各波段控制因素角度，研究光谱 变化的原因, 确定了不同生育时期洪涝胁迫特征波段和 植被指数。基于洪涝胁迫程度与稳定性较好的植被指数, 建立灾损评估模型。

洪涝胁迫后的变化不仅包括叶绿素、覆盖度还包括其 他理化指标以及土壤水分的变化，每个生育时期的情况

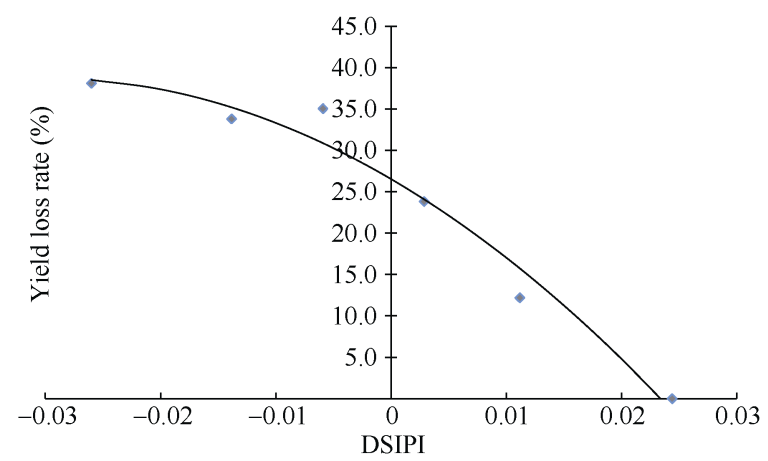

图 8 吐丝期玉米洪涝胁迫产量损失评估 DSIPI 模型拟合图 Fig. 8 Fitting chart of maize yield loss rate with DSIPI under flooding stress at silking stage 
各不相同, 还需进一步分析各指标与光谱变化的内在联 系, 尤其是适当的光谱预处理提取土壤背景含水量的数 据, 将有助于建立完善的遥感监测和评估技术体系。拔节 期、吐丝期洪涝胁迫与玉米遭受其他胁迫后光谱反射率的 变化趋势相同 ${ }^{[33]}$, 可辅助气象数据确定玉米是否遭受洪 涝胁迫。通常, 植株越健壮, 叶绿素吸收可见光进行光合 作用的能力和自我保护反射近红外光灼伤的能力越强, 可见光的反射率越低, 近红外反射率越高, 但灌浆期玉米 遭受胁迫后光谱呈现植株长势良好态势, 实质是玉米遭 受洪涝灾害贪青晚熟的表现。

试验过程中曾出现 2 次自然降雨, 对叶片叶绿素的持 续观测发现, 受光照和气温的影响, 降雨时叶片叶绿素急 剧下降, 雨后随着天气的好转, 叶绿素能够快速恢复到降 雨前水平。本试验光谱测试均于晴天开展, 对整体试验结 果无影响。

由遗传基因决定，不同品种有不同的耐涝特性，不同 土壤的渗水性、通气性亦不同, 本研究仅在红壤土试验地 用了目前试验区域主推品种之一, 代表性还不够全面。人 工模拟采用地下水, 水深为 $30 \mathrm{~cm}$, 不同于温度较高的自 然降雨, 且试验时天气为晴天而不是阴天, 试验条件下植 株和群体的变化与自然发生的洪涝胁迫还存在一些不同。 因此, 还需要进一步丰富受试品种与土壤类型, 继续开展 与自然洪涝胁迫更为吻合的试验。

拔节期、吐丝期和灌浆期的洪涝胁迫对玉米生长产生 了负面影响, 拔节期危害最大, 吐丝期次之, 灌浆期较轻, 这与王成业等人的研究结论一致 ${ }^{[33-34]}$ 。拔节期洪涝胁迫能 显著降低叶绿素的含量, 植株叶片发黄, 覆盖度急剧降低, 冠层光谱变为裸露土壤和胁迫后玉米植株的混合光谱, 可见光和近红外平台光谱反射率都显著降低。吐丝期洪涝 胁迫对叶绿素基本无影响，而覆盖度稍有降低，光谱曲线 变化不及拔节期显著。灌浆期洪涝胁迫稍微加速了叶绿素 的分解和叶片的衰老, 覆盖度略有下降, 但影响均不大。 可用与拔节期和吐丝期胁迫程度相关度都较高的结构不 敏感色素指数, 胁迫之后与胁迫前的差值 DSIPI 为参数建 立模型, 进行玉米洪涝灾害损失的评估。

\section{References}

[1] 滕腾. 中国玉米进出口贸易形势及影响因素分析. 南京农业 大学硕士学位论文, 江苏南京, 2015.

Teng T. Analysis on the Situation and Influence Factors of Chinese Maize Export and Import Trade. MS Thesis of Nanjing Agricultural University, Nanjing, Jiangsu, China, 2015 (in Chinese with English abstract).

[2] 中华人民共和国国家统计局. 中国统计年鉴. 北京: 中国统计 出版社，2019 [2020-03-01]. http://www.stats.gov.cn/tjjj/ndsj/ 2019/indexch.htm.

National Bureau of Statistics of the People's Republic of China. Citations format of China Statistical Yearbook. Beijing: China Statistics Publishing House, 2019. [2020-03-01]. http://www. stats.gov.cn/tjsj/ndsj/2019/indexch.htm (in Chinese).
[3] 潘瑞炽. 植物生理学. 北京: 高等教育出版社, 2012. pp 104-105.

Pan R Z. Plant Physiology. Beijing: Higher Education Press, 2012. pp 104-105 (in Chinese).

[4] 余卫东. 黄淮海地区涝渍胁迫对夏玉米的影响及时空变化研 究. 中国农业大学博士学位论文, 北京, 2014.

Yu W D. Research on the Effect of Waterlogging on the Growth and Yield of Summer Maize and Its Temporal-spatial Distribution in Huang-Huai-Hai Region. PhD Dissertation of China Agricultural University, Beijing, China, 2014 (in Chinese with English abstract).

[5] 周新国, 韩会玲, 李彩霞, 郭树龙, 郭冬冬, 陈金平. 拔节期 淹水玉米的生理性状和产量形成. 农业工程学报, 2014, 30(9): $119-125$.

Zhou X G, Han H L, Li C X, Guo S L, Guo D D, Chen J P. Physiological characters and yield formation of corn (Zea mays L.) under waterlogging stress in jointing stage. Trans CSAE, 2014, 30(9): 119-125 (in Chinese with English abstract).

[6] 梁哲军, 陶洪斌, 王璞. 淹水解除后玉米幼苗形态及光合生理 特征恢复. 生态学报, 2009, 29: 3977-3986.

Liang Z J, Tao H B, Wang P. Recovery effects of morphology and photosynthetic characteristics of maize (Zea mays L.) seedlings after waterlogging. Acta Ecol Sin, 2009, 29: 3977-3986 (in Chinese with English abstract).

[7] 任佰朝, 朱玉玲, 董树亭, 刘鹏, 赵斌, 张吉旺. 大田淹水对 夏玉米光合特性的影响. 作物学报, 2015, 41: 329-338.

Ren B C, Zhu Y L, Dong S T, Liu P, Zhao B, Zhang J W. Effects of waterlogging on photosynthetic characteristics of summer maize under field conditions. Acta Agron Sin, 2015, 41: 329-338 (in Chinese with English abstract).

[8] 杨京平, 陈杰. 不同生长时期土壤渍水对春玉米生长发育的 影响. 浙江农业学报, 1998, 10(4): 20-24.

Yang J P, Chen J. The effects of soil waterlogging at different growth stages on the growth and development of spring corn. Acta Agric Zhejiangensis, 1998, 10(4): 20-24 (in Chinese with English abstract).

[9] 刘祖贵, 刘战东, 肖俊夫, 南纪琴, 巩文军. 苗期与拔节期淹 涝抑制夏玉米生长发育、降低产量. 农业工程学报, 2013, 29(5): 44-52.

Liu Z G, Liu Z D, Xiao J F, Nan J Q, Gong W J. Waterlogging at seedling and jointing stages inhibits growth and development, reduces yield in summer maize. Trans CSAE, 2013, 29(5): 44-52 (in Chinese with English abstract).

[10] 郭庆法, 王庆成, 汪黎明. 中国玉米栽培学. 上海: 上海科学 技术出版社, 2004. p 47.

Guo Q C, Wang Q C, Wang L M. China Maize Cultivation. Shanghai: Shanghai Scientific and Technical Publishers, 2004. p 47 (in Chinese).

[11] Zaidi P H, Rafique S, Rai P K, Singh N N, Srinivasan G. Tolerance to excess moisture in maize (Zea mays L.): susceptible crop stages and identification of tolerant genotypes. Field Crops Res, 2004, 90: 189-202.

[12] Ren B Z, Zhang J W, Li X, Fan X, Dong S T, Liu P, Zhao B. Effects of waterlogging on the yield and growth of summer maize under field conditions. Can J Plant Sci, 2014, 94: 23-31.

[13] 孙忠翔, 唐培坤, 康尧强. 涝害对玉米生长发育的影响及应对 
措施. 现代化农业, 2014, (6): 7-8.

Sun Z X, Tang P K, Kang R Q. The effect of flood stress on maize's growth and countermeasures. Modern Agric, 2014, (6): 7-8 (in Chinese with English abstract).

[14] 浦瑞良, 宫鹏. 高光谱遥感及其应用. 北京: 高等教育出版社, 2000. pp 1-254.

$\mathrm{Pu}$ R L, Gong P. Hyperspectral Remote Sensing and Its Application. Beijing: Higher Education Press, 2000. pp 1-254 (in Chinese).

[15] 毕京佳. 基于遥感和 GIS 的洪水淹没范围估测与模拟研究. 中 国科学院研究生院(海洋研究所)硕士学位论文, 北京, 2016.

Bi J J. Estimation and Simulation of Flood Inundation Using Remote Sensing and GIS. MS Thesis of Institute of Oceanology, Chinese Academy of Sciences, Beijing, China, 2016 (in Chinese with English abstract).

[16] 苏亚丽. 耕地暴雨洪水灾害多源卫星遥感监测方法研究. 西 安科技大学硕士学位论文, 陕西西安, 2018.

Su Y L. Study on Remote Sensing Monitoring Method of Multi-source Satellite for Rainstorm and Flood in Farmland. MS Thesis of Xi'an University of Science and Technology, Xi'an, Shaanxi, China, 2018 (in Chinese with English abstract).

[17] 姜波, 孟灵, 邢前国. 2018 年夏季莱州湾南部寿光台风洪水受 害区遥感监测. 环境影响评价, 2019, 41(5): 83-87.

Jiang B, Meng L, Xing Q G. Monitoring of flood disaster footprints based on remote sensing: Shouguang floods of 2018, southern Laizhou Bay. Environ Impact Assess, 2019, 41(5): 83-87 (in Chinese with English abstract).

[18] 王一明. 干旱条件下基于 WOFOST 模型与遥感数据同化的玉 米产量模拟改进研究. 中国科学院大学(中国科学院遥感与数 字地球研究所)硕士学位论文, 北京, 2018.

Wang Y M. Improvement of Crop Yield Estimation under Drought Condition Based on WOFOST Model and Remote Sensing Data Assimilation. MS Thesis of Institute of Remote Sensing and Digital Earth, Chinese Academy of Sciences, Beijing, China, 2018 (in Chinese with English abstract).

[19] 靳华安, 王锦地, 柏延臣, 陈桂芬, 薛华柱. 基于作物生长模 型和遥感数据同化的区域玉米产量估算. 农业工程学报, 2012, 28(6): 162-173.

Jin H A, Wang J D, Bai Y C, Chen G F, Xue H Z. Estimation on regional maize yield based on assimilation of remote sensing data and crop growth model. Trans CSAE, 2012, 28(6): 162-173 (in Chinese with English abstract).

[20] 侯英雨, 王建林, 毛留喜, 宋迎波. 美国玉米和小麦产量动态 预测遥感模型. 生态学杂志, 2009, 28: 2142-2146.

Hou Y Y, Wang J L, Mao L X, Song Y B. Dynamic estimation models of corn and wheat yields in USA based on remote sensing data. Chin J Ecol, 2009, 28: 2142-2146 (in Chinese with English abstract).

[21] 赵英时. 遥感应用分析原理与方法. 北京: 科学出版社, 2013. pp 362-367.

Zhao Y S. The Principle and Method of Analysis of Remote Sensing Application. Beijing: Science Press, 2014. pp 362-367 (in Chinese).

[22] Richardson A J, Wiegand C L. Distinguishing vegetation from soil background information. Photogram Eng Remote Sens, 1977, 43: $1541-1552$.

[23] Rouse J W, Haas R H, Schell J A, Deering D W, Harlan J C. Monitoring the Vernal Advancement and Retrogradation of Natural Vegetation, NASAGSFC, Type I, Final Report, Greenbelt, MD, USA. 1974. p 371. http://www.doc88.com/p-60352201375 82.html.

[24] 刘良云. 高光谱遥感在精准农业中的应用研究. 中国科学院 遥感应用研究所博士学位论文, 北京, 2002.

Liu L Y. Applications of Hyperspectral Remote Sensing in Precision Agriculture. PhD Dissertation of Institute of Remote Sensing Applications, Chinese Academy of Sciences, Beijing, China, 2002 (in Chinese with English abstract).

[25] Singh R, Semwal D P, Rai A. Small area estimation of crop yield using remote sensing satellite data. Int J Remote Sens, 2002, 23: $49-56$.

[26] Penuelas J, Filella I, Gamon J A. Assessment of photosynthetic radiation-use efficiency with spectral reflectance. New Phytol, 1995, 131: 291-296.

[27] Lukina E V, Freeman K W, Wynn K J, Thomason W E, Mullen R W, Stone M L. Nitrogen fertilization optimization algorithm based on in-season estimates of yields and plant nitrogen uptake. Plant Nutr, 2001, 24: 885-898.

[28] Huete A R. A soil-adjusted vegetation index (SAVI). Remote Sens Environ, 1988, 25: 295-309.

[29] Haboudane D, Miller J R, Tremblay N, Zarco-Tejada P J, Dextraze $\mathrm{L}$. Integrated narrow-band vegetation indices for prediction of crop chlorophyll content for application to precision agriculture. Remote Sens Environ, 2002, 81: 416-426.

[30] Baret F, Guyot G. Potentials and limits of vegetation indices for LAI and APAR assessment. Remote Sens Environ, 1991, 35: 161-173.

[31] Penuelas J, Baret F, Filella I. Semiempirical indexes to assess carotenoids chlorophyll $a$ ratio from leaf spectral reflectance. Photosynthetica, 1995, 31: 221-230.

[32] 王利民, 刘佳, 邵杰, 杨福刚, 高建孟. 基于高光谱的春玉米 大斑病害遥感监测指数选择. 农业工程学报, 2017, 33(5): 170-177.

Wang L M, Liu J, Shao J, Yang F G, Gao J M. Remote sensing index selection of leaf blight disease in spring maize based on hyper spectral data. Trans CSAE, 2017, 33(5): 170-177 (in Chinese with English abstract).

[33] 王成业. 洪涝灾害对夏玉米生长发育及产量的影响. 河南农 业科学, 2010, (8): 20-21.

Wang C Y. The effect on growth and development and yield of summer maize. J Henan Agric Sci, 2010, (8): 20-21 (in Chinese with English abstract).

[34] 陈振, 梁守真, 王猛, 颜丙圈, 姚慧敏, 隋学艳, 王勇. 人工模 拟洪涝胁迫对夏玉米叶部性状及产量的影响. 山东农业科学, 2017, 49(4): 26-29.

Chen Z, Liang S Z, Wang M, Yan B T, Yao H M, Sui X Y, Wang Y. Effects of simulated flooding stress on yield and leaf characteristics of summer corn. Shandong Agric Sci, 2017, 49(4): 26-29 (in Chinese with English abstract). 